\title{
Nonradiative Energy Losses in Bulk-Heterojunction Organic Photovoltaics
}

\author{
Mohammed Azzouzi, ${ }^{1, \dagger}$ Jun Yan, ${ }^{1,2, \dagger}$ Thomas Kirchartz, ${ }^{3,4}$ Kaikai Liu, ${ }^{5}$ Jinliang Wang, ${ }^{5}$ Hongbin Wu, ${ }^{2}$ and Jenny Nelson ${ }^{1, *}$ \\ ${ }^{1}$ Department of Physics and Centre for Plastic Electronics, Imperial College London, \\ London SW7 2AZ, United Kingdom \\ ${ }^{2}$ Institute of Polymer Optoelectronic Materials and Devices, State Key Laboratory of Luminescent \\ Materials and Devices, South China University of Technology, Guangzhou 510640, China \\ ${ }^{3}$ IEK5-Photovoltaics, Forschungszentrum Jülich, 52425 Jülich, Germany \\ ${ }^{4}$ Faculty of Engineering and CENIDE, University of Duisburg-Essen, \\ Carl-Benz-Strasse 199, 47057 Duisburg, Germany \\ ${ }^{5}$ Beijing Key Laboratory of Photoelectronic/Electrophotonic Conversion Materials, \\ Beijing Institute of Technology, Beijing 100081, China
}

(Received 11 May 2018; revised manuscript received 20 July 2018; published 7 September 2018; corrected 15 April 2020)

\begin{abstract}
The performance of solar cells based on molecular electronic materials is limited by relatively high nonradiative voltage losses. The primary pathway for nonradiative recombination in organic donoracceptor heterojunction devices is believed to be the decay of a charge-transfer (CT) excited state to the ground state via energy transfer to vibrational modes. Recently, nonradiative voltage losses have been related to properties of the charge-transfer state such as the Franck-Condon factor describing the overlap of the CT and ground-state vibrational states and, therefore, to the energy of the CT state. However, experimental data do not always follow the trends suggested by the simple model. Here, we extend this recombination model to include other factors that influence the nonradiative decay-rate constant, and therefore the open-circuit voltage, but have not yet been explored in detail. We use the extended model to understand the observed behavior of series of small molecules:fullerene blend devices, where open-circuit voltage appears insensitive to nonradiative loss. The trend could be explained only in terms of a microstructure-dependent CT-state oscillator strength, showing that parameters other than CT-state energy can control nonradiative recombination. We present design rules for improving open-circuit voltage via the control of material parameters and propose a realistic limit to the power-conversion efficiency of organic solar cells.
\end{abstract}

DOI: 10.1103/PhysRevX.8.031055

\section{INTRODUCTION}

While the advent of nonfullerene acceptors has enabled a further efficiency increase for organic solar cells in the past two years [1,2], charge recombination is still a major impediment for further efficiency improvements. Fill factors of state-of-the-art organic solar cells seldom exceed $70 \%[3,4]$, which points at losses during charge-carrier collection. The open-circuit voltages have been increased substantially in recent years with peak values being in the

\footnotetext{
*Corresponding author. jenny.nelson@imperial.ac.uk

${ }^{\dagger}$ M. A., J. Y. contributed equally to this work.

Published by the American Physical Society under the terms of the Creative Commons Attribution 4.0 International license. Further distribution of this work must maintain attribution to the author(s) and the published article's title, journal citation, and DOI.
}

\author{
Subject Areas: Energy Research, Optoelectronics, \\ Physical Chemistry, \\ Semiconductor Physics
}

$1.0-1.1 \mathrm{~V}$ range for approximately $1.6-\mathrm{eV}$ band-gap absorbers [5]. Nevertheless, the open-circuit voltage losses in organic solar cells are still higher than in competing technologies such as lead-halide perovskites. Thus, research into the fundamental mechanisms and limitations for recombination in organic bulk heterojunction solar cells is urgently required. Recombination, in general, can be rate-limited either by transport of charge carriers towards each other or by dissipation of the energy of the polaron pair, which is believed to occur via a so-called interfacial charge-transfer (CT) state in organic heterojunction solar cells $[6,7]$. These two processes of transport and energy dissipation have to happen in series, and therefore the slower of the two processes is the rate-limiting process. So far, models of recombination in organic solar cells have mostly either assumed that the diffusion is the rate-limiting step [8] or, if they have included an additional dissipation step, the decay coefficient was usually considered a fit parameter $[9,10]$. The discussion on the origin and the 
fundamental understanding of the decay coefficient of a charge-transfer state has only recently been moved into the focus of the community's attention [11]. Recent work by Benduhn et al. [12] shows that the open-circuit voltage loss due to nonradiative recombination changes with the energy of the charge-transfer state over a large set of different donor-acceptor combinations. This change can be rationalized by understanding that a higher overlap of the vibrational modes of the charge transfer and ground state increases the rate of recombination. With the energy of the highest-energy vibrational modes being essentially the same for all organic semiconductors, it is then the amount of energy lost in the recombination event (i.e., the energy of the CT state) that dominates the rate of the transition. This result is essentially the so-called "energy-gap law" discussed by a range of authors in the past for both relaxation processes in molecules [13-15] and recombination processes in inorganic semiconductors [16-19]. The importance of the mechanism of nonradiative recombination for the performance of organic photovoltaic (OPV) devices arises partly from the high vibrational energy of the carboncarbon bonds that allows a significant spacing of the vibrational states.

The model in Ref. [12] focuses solely on the effect of the energy of the CT state and neglects other properties of the transition. This approach can reproduce the overall trend with the change of $E_{\mathrm{CT}}$, but it cannot explain the significant scatter around the trend in the experimental data.

In an attempt to understand the effect of the characteristics of the charge-transfer state on the recombination rates, we establish a model to quantify the nonradiative voltage losses $\left(\Delta V_{\text {oc,nr }}\right)$. For bulk heterojunction OPV devices, based on the fact that both radiative and nonradiative recombination occur through the lowest-energy charge-transfer state. The model incorporates the effects of properties of the CTstate-to-ground-state transition, such as the reorganization energies, oscillator strength, difference in Gibbs free energy, and energy of the vibrational states, on $\Delta V_{\text {oc,nr }}$. As well as quantifying the nonradiative voltage losses, we express the absorption rate using a similar approach to that used for the radiative recombination. Using the absorption rate, we can calculate the absorption coefficient and therefore reconstruct the external quantum efficiency (EQE) of the devices assuming perfect charge-collection efficiency. The reconstructed EQE is used to estimate the radiative open-circuit voltage $V_{\text {oc,rad }}$. Combining both the calculated $V_{\text {oc,rad }}$ and $\Delta V_{\text {oc,nr }}$, we can estimate the open-circuit voltage of the cell: $V_{\text {oc }}=V_{\text {oc,rad }}-\Delta V_{\text {oc,nr }}$.

We apply this model to explain the trend in open-circuit voltage upon changing the solvent-vapor annealing (SVA) time of different small molecule-fullerene devices. We observe that both the nonradiative voltage loss and $V_{\text {oc,rad }}$ increase with the SVA time. These two effects compensate each other, resulting in a nearly unchanged value of the open-circuit voltage. This trend is the opposite of that expected from Ref. [12] and could be explained within the proposed model only by a decrease of the oscillator strength of the transition with an increasing SVA time. In the framework of the model, we evaluate different strategies for enhancing the open-circuit voltage considering a set of CT state properties and their effect on both radiative and nonradiative losses. Using this approach, we explore the limits to the power-conversion efficiencies of OPV devices, considering the limitations incurred by this recombination process.

\section{THEORY}

\section{A. Open-circuit voltage-loss analysis}

An ideal solar cell, as presented by Shockley and Queisser (SQ) [20], has a steplike absorptance with the band gap being the energy of the step. In addition, in the Shockley-Queisser limit, all generated electron-hole pairs are collected, and the only recombination process is radiative recombination. For real devices, the $V_{\mathrm{oc}}$ is further reduced by a broadening of the absorption edge compared to the ideal step function assumed in the SQ limit and by nonradiative recombination. We differentiate the nonradiative and the absorption-broadening losses the same way as presented by Yao et al. [21]. First, we define the radiative open-circuit voltage as

$$
q V_{\mathrm{oc}, \mathrm{rad}}=k_{B} T \ln \left(\frac{J_{\mathrm{ph}}\left(V_{\mathrm{oc}}\right)}{J_{0, \mathrm{rad}}}+1\right)
$$

where $J_{0, \text { rad }}$ is the saturation current density calculated by considering only the blackbody radiation of the cell with the real absorption profile, $k_{B}$ Boltzmann's constant, $T$ the temperature of the sample, $q$ the elementary charge and $J_{\mathrm{ph}}$ $\left(V_{\text {oc }}\right)$ the photocurrent at open-circuit condition. We then define the voltage losses due to the smeared-out absorption edge as

$$
\Delta V_{\mathrm{oc}, \mathrm{abs}}=V_{\mathrm{oc}, \mathrm{SQ}}-V_{\mathrm{oc}, \mathrm{rad}}
$$

and the nonradiative voltage losses via

$q \Delta V_{\mathrm{oc}, \mathrm{nr}}=q V_{\mathrm{oc}, \mathrm{rad}}-q V_{\mathrm{oc}}=-k_{B} T \ln \left[Q_{\mathrm{LED}}\left(V_{\mathrm{oc}}\right)\right]$.

Here, $Q_{\mathrm{LED}}\left(V_{\mathrm{oc}}\right)$ is the external quantum efficiency of the electroluminescence (EL) emission at an applied internal voltage equal to $V_{\text {oc }}$ of the cell. For a thin-film solar cell, where a photon emitted by radiative recombination is either emitted with an emission probability $p_{e}$ or reabsorbed with a probability $p_{r}$ [22], we can relate $Q_{\mathrm{LED}}\left(V_{\mathrm{oc}}\right)$ to the internal quantum yield of the EL emission $\left(\mathrm{IQ}_{\mathrm{LED}}\right)$ if we consider perfect charge extraction (i.e., no surface recombination) [23] and recombination rates independent of the spatial position in the absorber layer via 


$$
Q_{\mathrm{LED}}\left(V_{\mathrm{oc}}\right)=\frac{p_{e} \mathrm{IQ}_{\mathrm{LED}}\left(V_{\mathrm{oc}}\right)}{1+\left(p_{e}-1\right) \operatorname{IQ}_{\mathrm{LED}}\left(V_{\mathrm{oc}}\right)} .
$$

Here, the internal luminescence quantum efficiency

$$
\mathrm{IQ}_{\mathrm{LED}}\left(V_{\mathrm{oc}}\right)=\frac{K_{r}}{K_{r}+K_{\mathrm{nr}}}
$$

is defined as the ratio of the rate constant of radiative decay from the CT state to the ground state, $K_{r}$, and the total decay-rate constant, which is the sum of $K_{r}$ and the nonradiative recombination-rate constant $K_{\mathrm{nr}}$. We calculate the emission probability by estimating the absorption coefficient and absorptance using an expression for the absorption rate and using Eq. (6) in Ref. [24]. Details about these calculations are presented in the optical-model section. We can then express the nonradiative voltage losses as

$$
q \Delta V_{\mathrm{oc}, \mathrm{nr}}=-k_{B} T \ln \left(\frac{p_{e} K_{r}}{K_{\mathrm{nr}}+p_{e} K_{r}}\right) .
$$

\section{B. Recombination pathways}

In this model, we consider only recombination that occurs at the CT state of the donor-acceptor complex, i.e., radiative recombination of the $\mathrm{CT}$ state and the nonradiative relaxation of the CT state to the ground state, as illustrated in Fig. 1. The CT state can be generated either during the charge-separation process of the exciton that results from optical excitation or when the charge carriers meet at a donor-acceptor interface.
To calculate the rates of recombination, we represent the solar cell by a two-state model consisting of a ground state and a CT state similar to the model in Ref. [12]. The nonradiative recombination-rate constant will be enhanced by the overlap of the occupied vibrational mode of the CT state with the most energetically accessible vibrational modes of the ground state, while the radiative recombination is due to spontaneous emission from the chargetransfer state to any vibrational mode of the ground state. In molecular structures containing many carbon-carbon bonds, the vibrational spacing energy $\hbar \Omega$ is typically in the region of $0.15 \mathrm{eV}$.

\section{Recombination rates}

Radiative and nonradiative electron transfer from the excited CT state to the ground state in a donor-acceptor complex are described classically by the energy-gap law [25-27]. We apply the same approach that has been used for donor-acceptor complexes in solution to quantify the recombination rates from the $\mathrm{CT}$ states in the absorber layer of a solid-state donor-acceptor blend. This approach is similar to the one adopted by Benduhn et al. to calculate the recombination rates. Here, we adopt the method introduced by Jortner [28], where we consider the solvent modes to be thermally activated while the molecular modes are frozen. This method means that we effectively consider the surrounding molecular environment as a solvent for the CT state.

Using this assumption, the nonradiative rate constant $\left(K_{\mathrm{nr}}\right)$ can be described, by the electron transfer-rate constant expression using Fermi's golden rule and the BornOppenheimer approximation, as a product of the electronic coupling $V$ between the CT state and ground state and the Franck-Condon weighted density of states $\operatorname{FCWD}(\hbar \omega)$ : (a)

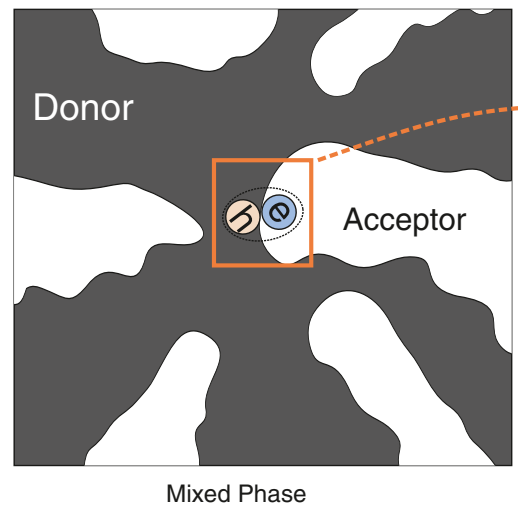

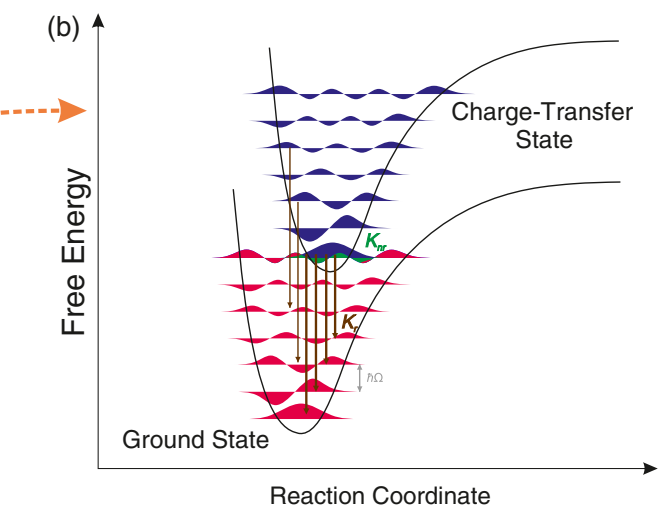

FIG. 1. Recombination mechanism in organic photovoltaics. (a) Schematic illustrating an electron and hole occupying an interfacial charge-transfer state in a bulk-heterojunction system. (b) The potential energies of the ground state and charge-transfer state at the donoracceptor interface as a function of the reaction coordinate. The quantized vibrational modes are shown as the colored waves: red for modes of the electronic ground state and blue for modes of the charge-transfer state. The green overlap between the lowest vibrational state of the charge-transfer state (which defines the energy of the apparent charge-transfer state) and a vibrationally excited ground state indicates the nonradiative recombination pathway. The arrows depict possible radiative decay pathways. While we indicate here some radiative decay from higher-energy vibronic modes, most decay originates from the lowest vibronic state of the charge-transfer state. 


$$
K_{\mathrm{nr}}=\frac{2 \pi}{\hbar} V^{2} \mathrm{FCWD}(0),
$$

where $\hbar$ is the reduced Planck constant in $\mathrm{eV} \mathrm{s}$. The Franck-Condon weighted density of states accounts for transitions between all vibrational modes of the initial (CT) state and all vibrational modes of the final (ground) state where the states differ in energy by $\hbar \omega$ [Eq. (S4) [29]]. For nonradiative decay, it takes the argument 0 . In general,

$$
\operatorname{FCWD}(\hbar \omega)=\frac{1}{\sqrt{4 \pi \lambda_{l} k_{B} T}} \sum_{w=0}^{\infty} \sum_{t=0}^{\infty} \frac{e^{-S} S^{w-t} t !}{w !}\left[L_{t}^{w-t}(S)\right]^{2} e^{-\left\{\left[\hbar \omega-\Delta G_{0}+\lambda_{l}+(w-t) * \hbar \Omega\right]^{2} / 4 \lambda_{l} k_{B} T\right\}} e^{-t \hbar \Omega / k_{B} T}
$$

with $\lambda_{l}$ the low-frequency reorganization energy, $S=$ $\left(\lambda_{v} / \hbar \Omega\right)$ is the Huang Rhys factor [30], and $\lambda_{v}$ and $\Omega$ are, respectively, the reorganization energy and the harmonic frequency of the quantized high-frequency mode (assumed the same for all vibronic states). $\Delta G_{0}$ is the difference in Gibbs free energy between the two states. $w$ and $t$ designate the quantum number of the vibrational modes of the ground state and the CT state, respectively, and $L_{t}^{w-t}(S)$ is the generalized Laguerre polynomial of degree $t[31,32]$. In this work, we consider the contribution of the many vibrational modes of the initial state with a Boltzmann distribution. The high-frequency reorganization energy $\left(\lambda_{v}\right)$ is often related to the inner-sphere reorganization energy, which is attributed to the energy cost associated with the geometry change in the donoracceptor complex from the optimized geometry in the ground state and its optimized geometry in the chargetransfer state [33]. The inner-sphere reorganization energy can be estimated using a density-functional-theory calculation on the donor-acceptor complex [34]. The lowfrequency reorganization energy $\left(\lambda_{l}\right)$, on the other hand, is associated with reorganization of the nuclear and dielectric environment to accommodate the charge-transfer event as well as the low-frequency modes of the donor-acceptor complex itself. If the two reorganization energies have been commonly defined for small molecular systems in solution as the inner reorganization energy for the high-frequency one and the outer reorganization energy for the low-frequency one, this assumption is not always valid for larger molecules in a solid state; in fact, it has been shown that the geometry change on the donoracceptor complex related to the CT-state transition contributes in a large part to the value of the low-frequency mode reorganization energy [35]. Therefore, both values are strongly dependent on the chemical structure of the materials.

Using the same approach, we can express the radiative rate constant using the operator $O=\left(q / m_{e} c\right) \vec{A} \vec{p}$, where $c$ is the speed of light in vacuum, $m_{e}$ the electron rest mass, where $\vec{A}$ is the vector potential of the electromagnetic field and $\vec{p}$ the momentum operator. Using the dipole approximation, the radiative rate constant per photon energy can be expressed using the transition dipole moment [18] as

$$
k_{r}(\hbar \omega)=\frac{1}{3 \pi \epsilon_{0} \hbar^{4}}\left(\frac{\hbar \omega}{c}\right)^{3} M^{2} \mathrm{FCWD}(\hbar \omega)
$$

where $\epsilon_{0}$ is the permittivity of free space. The net radiative recombination-rate constant $K_{r}$ from the CT state to the ground state (GS) is the sum of contributions $k_{r}(\hbar \omega)$ over all the photon energies $\hbar \omega$ :

$$
K_{r}=\int k_{r}(\hbar \omega) d \hbar \omega
$$

Considering that the optical transition is an adiabatic transition, we express the transition dipole moment $M$ in terms of the oscillator strength $f_{\text {osc }}$ and the average frequency of the emitted photon $\omega_{\text {avg }}$ [36] via

$$
M^{2}=\frac{3}{2} \frac{\hbar^{2}}{\hbar \omega_{\mathrm{avg}} m_{e}} f_{\mathrm{osc}}
$$

This expression helps by relating the transition dipole moment to parameters we can calculate using the time-dependent density-functional theory such as the oscillator strength of the charge-transfer state and the average frequency of the emitted photon [37]. Moreover, we relate the electronic coupling to the transition dipole moment using the generalized MullikenHush (GMH) method [38]. This method is commonly used to estimate the transition elements of diabatic transitions using adiabatic elements [39]. The method is based on the definition of adiabatic states as the states with a transition dipole moment normal to the direction $(\Delta \vec{\mu} /|\Delta \vec{\mu}|) \Delta \vec{\mu}=\overrightarrow{\mu_{\mathrm{CT}}}-\overrightarrow{\mu_{\mathrm{GS}}}$, where $\overrightarrow{\mu_{\mathrm{CT}}}$ and $\overrightarrow{\mu_{\mathrm{GS}}}$ are the static dipole moment of the CT state and the GS, respectively. This assumption leads to the generalized expression for the electronic coupling

$$
V=\frac{M E_{\mathrm{CT}}}{\sqrt{\mid \Delta \overrightarrow{\mu \mid}^{2}+4 M^{2}}}
$$

Using the established equations (6), (7), (10), (11), and (12), we express the nonradiative voltage loss $\Delta V_{\mathrm{oc}, \mathrm{nr}}$ as a function of the different characteristics of the 
charge-transfer state. As a result, $\Delta V_{\text {oc.nr }}$ depends on the difference in Gibbs free energy between the two states $\Delta G_{0}$, the low- and high-frequency reorganization energies $\left(\lambda_{l}, \lambda_{v}\right)$, the difference in the static dipole moment between the two states $|\Delta \vec{\mu}|$, the oscillator strength $f_{\text {osc }}$ of the CT state, and the vibrational energy $\hbar \Omega$. Thus, $\Delta V_{\text {oc,nr }}=f\left(\Delta G_{0}, \lambda_{l}\right.$, $\left.\lambda_{v}, f_{\text {osc }},|\Delta \vec{\mu}|, \hbar \Omega\right)$. Considering the common case for organic devices where the nonradiative rate constant is orders of magnitude higher than the radiative one $\left(K_{\mathrm{nr}} \gg K_{r}\right), \Delta V_{\mathrm{oc}, \mathrm{nr}}$ is simplified to

$$
\Delta V_{\mathrm{oc}, \mathrm{nr}}=-k_{B} T \ln \left(p_{e} \frac{1}{3 \pi \epsilon_{0} \hbar^{4}} \frac{\left(\mid \Delta \overrightarrow{\left.\mu\right|^{2}}+6 \frac{\hbar^{2}}{\hbar \omega_{\text {avg }} m_{e}} f_{\mathrm{osc}}\right) \int \operatorname{FCWD}(\hbar \omega)(\hbar \omega)^{3} d \hbar \omega}{\frac{2 \pi}{\hbar} \operatorname{FCWD}(0) E_{\mathrm{CT}}^{2}}\right) .
$$

\section{Optical model for EQE reproduction}

We use the same approach used for the radiative recombination-rate constant (i.e., rate of spontaneous emission) to calculate the rate constant of photon absorption. To promote an electron from the ground state to the charge-transfer state, the spectral absorption rate can be expressed using the same approach, but we use a factor $N$ to account for the density of photons in the considered volume:

$$
k_{r, \mathrm{abs}}(\hbar \omega)=\frac{1}{3 \pi \epsilon_{0} \hbar^{4}}\left(\frac{\hbar \omega}{c}\right)^{3} M^{2} \mathrm{FCWD}_{\mathrm{abs}}(\hbar \omega) N
$$

Here, the Franck-Condon weighted density-of-states factor accounts for upward transitions from vibrational modes of the ground state to modes of the CT state, assuming Boltzmann statistics for the initial state occupation:

$$
\mathrm{FCWD}_{\mathrm{abs}}(\hbar \omega)=\frac{1}{\sqrt{4 \pi \lambda_{l} k_{B} T}} \sum_{w=0}^{\infty} \sum_{t=0}^{\infty} \frac{e^{-S} t ! S^{w-t}}{w !}\left[L_{t}^{w-t}(S)\right]^{2} e^{-\left\{\left[-\hbar \omega+\Delta G_{0}+\lambda_{l}+(w-t) \hbar \Omega\right]^{2} / 4 \lambda_{l} k_{B} T\right\}} e^{-t \hbar \Omega / k_{B} T}
$$

Here, $w$ and $t$ designate, respectively, the quantum number of the vibrational modes of the final and initial state. Using the photogeneration rate, we calculate the Einstein coefficient $B$ for the stimulated absorption [40]. [The Einstein coefficient $B$ is defined as $k_{r \text {,abs }}(\hbar \omega)=B \rho$, where $\rho$ is the total energy in the field per unit volume between the frequencies $\omega$ and $\omega+d \omega$. It represents the transition rates in such a way that the field-dependent effect proportional to $N$ is separated from the field-independent effects.] We relate $B$ to the absorption coefficient, using the established formula [41], if we consider a uniform distribution of the site with a volume $\mathcal{V}$ and find

$$
\alpha(\hbar \omega)=\frac{\frac{n}{c} B \hbar \omega}{\mathcal{V}}=\frac{n}{c} \frac{1}{6 \epsilon_{0} \hbar} M^{2} \mathrm{FCWD}_{\mathrm{abs}}(\hbar \omega) \hbar \omega \frac{1}{\mathcal{V}},
$$

where $n$ is the refractive index of the blend. For the overall absorption coefficient, we consider both the contribution of the charge-transfer state and the exciton state. We can express both with Eq. (16) using different parameters for the two states. For the exciton state, we consider similar parameters as for the CT state, apart from the value of $\Delta G_{0}$ that becomes $E_{G}$ instead of $E_{\mathrm{CT}}$ and an oscillator strength value of $f_{\text {osc }}=5$, which is at least 2 orders of magnitude higher than the one of the CT state. We can also take into account the CT-state density by changing the distribution of interfacial states compared to excitonic states. For this purpose, we introduce $R_{\mathrm{CT} / E}$ to represent the ratio of $\mathrm{CT}$ states compared to the excitonic states $\left(\mathcal{V}_{\mathrm{CT}}=\left(\mathcal{V}_{E} / R_{\mathrm{CT}, E}\right)\right)$. In this work, we consider $\mathcal{V}_{E}=1 \AA^{3}$. On top of the contribution of these two states, we consider that, for energies above $E_{g}+2 k_{B} T, \alpha$ follows the square-root law of a direct semiconductor:

$\alpha(\hbar \omega)= \begin{cases}\alpha_{\mathrm{CT}}+\alpha_{\mathrm{ex}} & \text { for } \hbar \omega<E_{g}+2 k_{B} T, \\ \alpha_{0} \times \sqrt{\frac{\hbar \omega-E g}{k_{B} T}} & \text { for } \hbar \omega>E_{g}+2 k_{B} T .\end{cases}$

For simplicity, we choose $\alpha_{0}=(2 / d)$, where $d$ is the thickness of the device, to account for the strong absorption of the high-energy photons within the film thickness. Using the absorption coefficient given by Eq. (17), we can express the absorptance $[A(E)]$, assuming a Beer-Lambert law without interference, a zero reflectance at the surface, and a unity reflectance from the back electrode, and obtain

$$
A(E)=1-\exp [-2 d \alpha(E)] .
$$

Here, $d$ is the thickness of the device. Assuming perfect electron transport and no losses of photoinduced charges, we can consider the absorptance as the ideal EQE of the 
device. This assumption allows us to calculate the radiative open-circuit voltage

$$
q V_{\mathrm{oc}, \mathrm{rad}}=k_{B} T \ln \left(\frac{J_{\mathrm{ph}}\left(V_{\mathrm{oc}}\right)}{\int A(E) \phi_{b b}(E) d E}+1\right) .
$$

We finally calculate the emission probability using the expression [24]

$$
p_{e}=\frac{\int A(E) \phi_{\mathrm{bb}}(E) d E}{4 d \int n^{2} \alpha(E) \phi_{\mathrm{bb}}(E) d E} .
$$
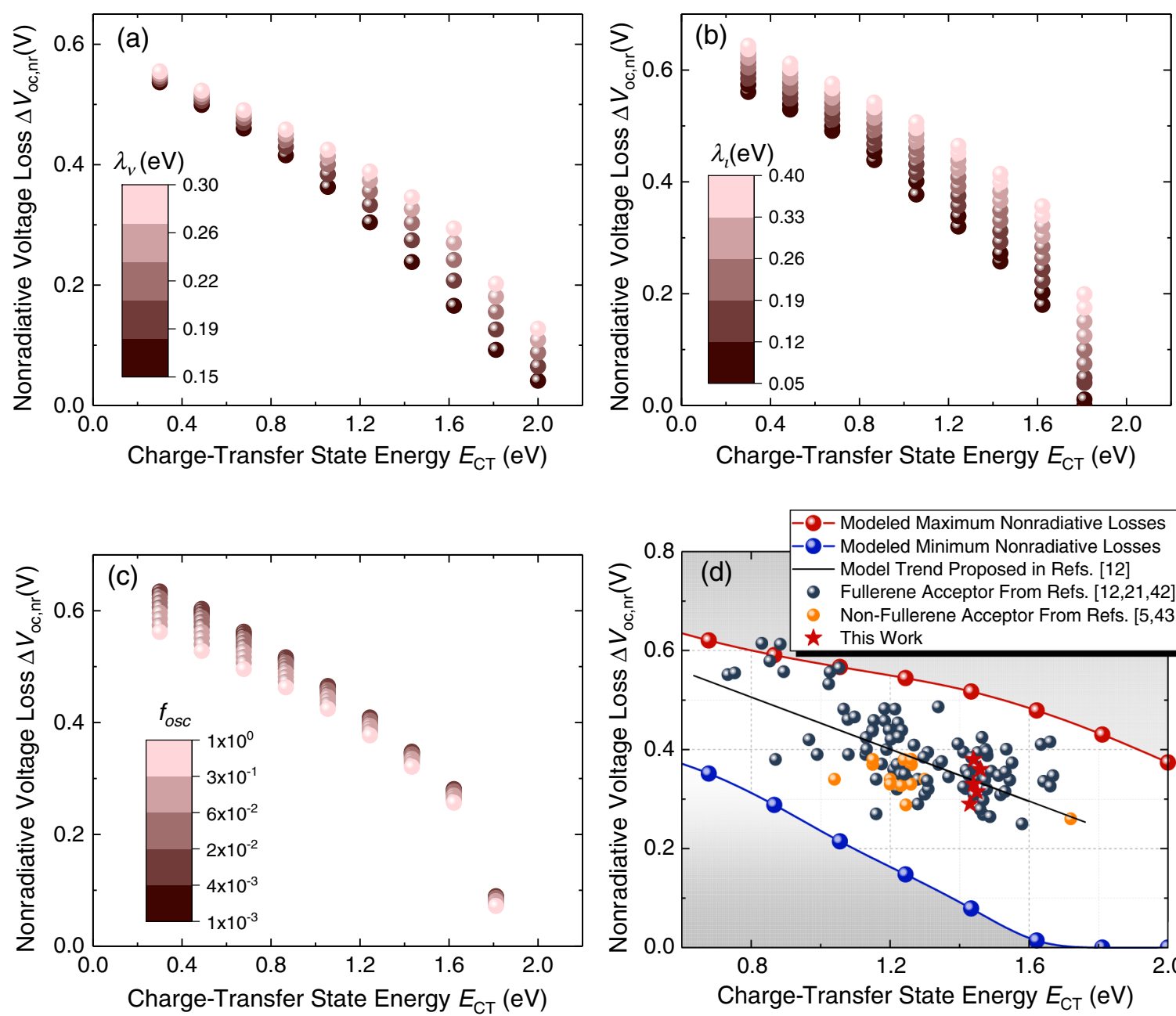

FIG. 2. Nonradiative voltage loss as a function of the energy of the charge-transfer state with respect to changed (a) high-frequency reorganization energy $\lambda_{v}$, (b) low-frequency reorganization energy $\lambda_{l}$, and (c) oscillator strength $f_{\text {osc }}$. (d) Predicted maximum (red line) and minimum (blue line) nonradiative voltage losses $\Delta V_{\mathrm{oc}, n \mathrm{n}}$ as a function of the energy of charge-transfer state $E_{\mathrm{CT}}$ obtained using the proposed model sampling a range of values for each of the six parameters. The key parameters for modeling the "maximum" and "minimum" cases are $\lambda_{v}=0.32 \mathrm{eV}, \lambda_{l}=0.4 \mathrm{eV}, f_{\text {osc }}=4 \times 10^{-3}$ and $\lambda_{v}=0.15 \mathrm{eV}, \lambda_{l}=0.05 \mathrm{eV}, f_{\text {osc }}=4 \times 10^{-1}$, respectively. For comparison, a variety of reported experimental values from the literature are shown (circular symbols) [5,12,21,42-44]. The data labeled "this work" is obtained from BIT-4F: $\mathrm{PC}_{71} \mathrm{BM}$ devices in the experimental section. A complicated version of (d) specifying the references can be found in Fig. S1 [29]. 
average for the values of $\Delta V_{\mathrm{oc}, \mathrm{nr}}$, where we explicitly vary only the parameter of interest and $\Delta G_{0}$ and sample all other parameters within the reported ranges. This approach helps quantify the average impact of the different parameter on $\Delta V_{\text {oc,nr }}$ for a fixed $\Delta G_{0}$. Henceforth, we consider $E_{\mathrm{CT}}=$ $\Delta G_{0}$ in order to be able to compare these data to experimental results. This approximation neglects the effect of the entropy as is commonly done.

The first three graphs in Figs. 2(a)-2(c) show a strong dependence of $\Delta V_{\mathrm{oc}, \mathrm{nr}}$ on $E_{\mathrm{CT}}$ and a significant dependence on the other parameters. Figures 2(a) and 2(b) show that the reorganization energies have a significant effect on $\Delta V_{\text {oc,nr }}$ at constant $E_{\mathrm{CT}}$. The high-frequency reorganization energy has a smaller effect at lower energies, while at $\mathrm{CT}$ energies closer to typical values of $E_{\mathrm{CT}}$ variation in $\lambda_{v}$ changes $\Delta V_{\mathrm{oc}, \mathrm{nr}}$ by $0.1-0.2 \mathrm{~V}$. The effect of $\lambda_{l}$ is less sensitive to $E_{\mathrm{CT}}$, with a stronger effect than $\lambda_{v}$ due to the wider range considered. The oscillator strength appears to have a smaller effect on the mean value of $\Delta V_{\text {oc,nr }}$ due to the fact that its effect would be less significant for high values of the difference in the static dipole moment. In fact, from the GMH formula it appears that both values are entangled.

Figure 2(d) shows the span of $\Delta V_{\text {oc,nr }}$ as a function of $E_{\mathrm{CT}}$ resulting from the parameter exploration compared to experimental data extracted from Refs. [5,12,21,42-44] as well as from the results of the present study. Most of the experimental data sit inside the calculated range for $\Delta V_{\mathrm{oc}, \mathrm{nr}}$, which supports the idea that the model can predict realistic values for the nonradiative voltage losses. The model predicts lower nonradiative losses than the currently achieved values; the closest point to the lower limit predicted by the model lies around $0.1 \mathrm{eV}$ higher than the predicted minimum at that CT energy. This result shows that there is an improvement to be made in this respect and that a higher $V_{\text {oc }}$ can still be achieved with organic photovoltaic technology. This analysis rests on the idea that additional loss pathways such as nonradiative recombination via the contacts and disorder in the CT-state energy can be avoided.

\section{CASE STUDY OF MULTIFLUORINE- SUBSTITUTED SMALL MOLECULES UNDER SOLVENT-VAPOR ANNEALING}

The results in the previous section emphasize that $\Delta V_{\text {oc,nr }}$ depends strongly on different parameters of the charge-transfer state. The large number of parameters considered in the model makes it hard to pinpoint which parameter induces the change in the open-circuit voltage losses for any organic solar cell. However, for a system where we limit the number of variables, we can aim to explain what controls the change in $\Delta V_{\text {oc,nr }}$.

For a bulk heterojunction device, the donor-acceptor phase separation determines the properties of the interfacial area and, hence, the properties of the CT states. SVA has attracted attention recently, mainly due to the capability of changing donor-acceptor phase separation and the device performance in small molecule-fullerene organic solar cells, which makes SVA a useful technique to understand the effect of changing CT-state microscopic properties.

Recently, a family of linear donor-acceptor multifluorine-substituted oligomers (BIT series) was developed: BIT-4F, BIT-6F, BIT-8F, and BIT-10F [45], which are all sensitive to SVA post treatment, making them good targets for this study. The main difference in the structure of these oligomers is their chain length, as shown in Fig. S2 [29]. Therefore, we can consider that the reorganization energies of the charge-transfer state would not change significantly. Exposing films of these oligomers blended with $\mathrm{PC}_{71} \mathrm{BM}$ to $\mathrm{CH}_{2} \mathrm{Cl}_{2}$ vapor increases the phase separation in the blend and increases the crystalline content of the film as reported in Refs. $[45,46]$. The change of the interface properties is believed to affect the properties of the charge-transfer state [47]. For these reasons, we use this family of oligomers with different exposure times to $\mathrm{CH}_{2} \mathrm{Cl}_{2}$ vapor to investigate the effect of the change of the interface properties on $\Delta V_{\text {oc,nr }}$.

The extracted device-performance data from currentvoltage characteristics are summarized in Figs. S3-S6 [29]. As clearly shown, BIT-4F with the shortest chain length shows the most sensitive response upon SVA, whereas devices based on molecules with longer chains, i.e., BIT-8F and BIT-10F based, stay relatively unaffected. The decrease in short-circuit current density $\left(J_{\mathrm{sc}}\right)$ is mainly caused by an enlarged donor-acceptor phase separation and the concomitant inefficient exciton diffusion and charge transfer $[46,48]$. The fill factor (FF) shows an expected fast increase at short SVA times and a slow drop after a certain time threshold. The overall power-conversion efficiency (PCE) presents a similar trend as $J_{\text {sc }}$. Interestingly, the opencircuit voltage $\left(V_{\text {oc }}\right)$ remains almost constant around $0.9 \mathrm{~V}$, especially for BIT-8F and BIT-10F with longer chains, despite a small drop for a very short exposure time in the case of BIT-4F and BIT-6F.

We should also emphasize here that changing the donoracceptor domain size will potentially change the properties of interfacial charge-transfer states as well as their interface area. Aiming to understand the $V_{\text {oc }}$ trend, we carry out a detailed energy-loss analysis. We measure the external quantum efficiency and the electroluminescence of blend devices made from the four oligomers with different exposure times to the solvent vapor. Figure 3(a) presents the EQE and EL data for BIT-4F with different SVA times, as well as the extended EQE constructed using the EL data by the same approach as Ref. [21]. Upon increasing the exposure time, the absorption edge of the EQE gets sharper. For the EL spectra, we mainly notice a decrease of the relative intensity of the first peak upon the SVA time without a significant change in the peak energy of the first peak. The small change in the energy of the first peak could 

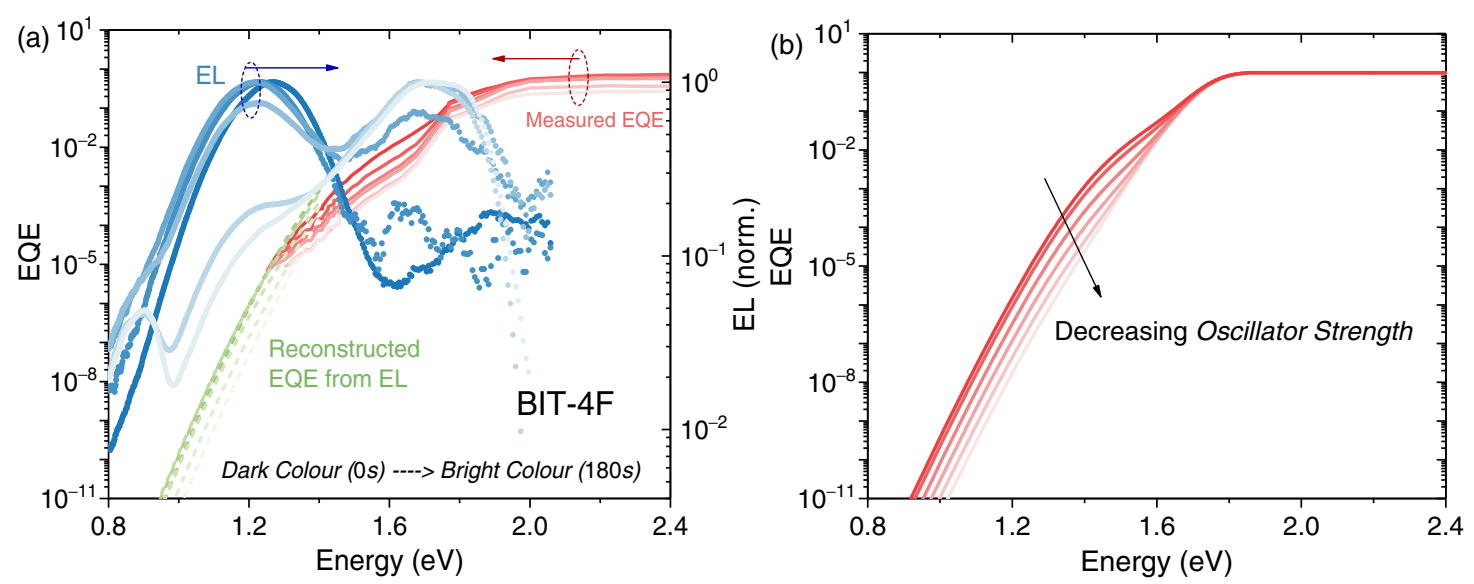

FIG. 3. (a) EL and EQE for BIT-4F:PC71BM blend devices with different SVA processing times. The external quantum efficiencies are composed of the directly measured EQE (red solid lines) and the EQE determined from the EL spectra (blue dashed lines). (b) Reproduced EQE spectra based on the optical model derived above. The different curves are obtained by changing only the oscillator strength of the charge-transfer state, keeping the properties of the excitonic state the same.

be interpreted as a relatively unchanged energy of the lowest charge-transfer state. The same observations are valid for all four oligomers (Fig. S7 [29]), with a less noticeable effect for the larger molecules. This result agrees with the fact that SVA induces fewer changes in the morphology of the larger-molecule blends.

Using the extended EQE for the four oligomers in [Figs. 3(a) and S7 [29]], we calculate $V_{\text {oc,rad }}$ and $\Delta V_{\text {oc,nr }}$ using the method in Ref. [21]. The results are presented in Fig. 4, where we plot $\Delta V_{\mathrm{oc}, \mathrm{nr}}$ against $V_{\mathrm{oc}, \mathrm{rad}} . \Delta V_{\mathrm{oc}, \mathrm{nr}}$ and $V_{\text {oc,rad }}$ increase by approximately the same amount with the SVA time, and, given that $V_{\mathrm{oc}}$ is defined as $V_{\mathrm{oc}, \mathrm{rad}}-$ $\Delta V_{\mathrm{oc}, \mathrm{nr}}$, this result agrees perfectly with the small changes observed in the $V_{\mathrm{oc}}$ of the devices. However, since the band gap of the absorber does not change upon SVA, these results suggest that the losses due to the broadening of the absorption peak decrease, whereas the nonradiative losses increase. Since the two losses are believed to happen due to recombination at the same interfaces, the fact that $\Delta V_{\mathrm{oc}, \mathrm{nr}}$ and $\Delta V_{\text {oc,rad }}$ compensate each other is counterintuitive. In fact, if we explain the decreasing $q \Delta V_{\text {oc,abs }}$ with a decreased density of interfacial sites, $\Delta V_{\text {oc,nr }}$ should not be affected, as it is mainly the ratio between radiative and nonradiative rates of recombination.

According to our model, an increase in $V_{\text {oc,rad }}$ can be related to either a change in the energy of the
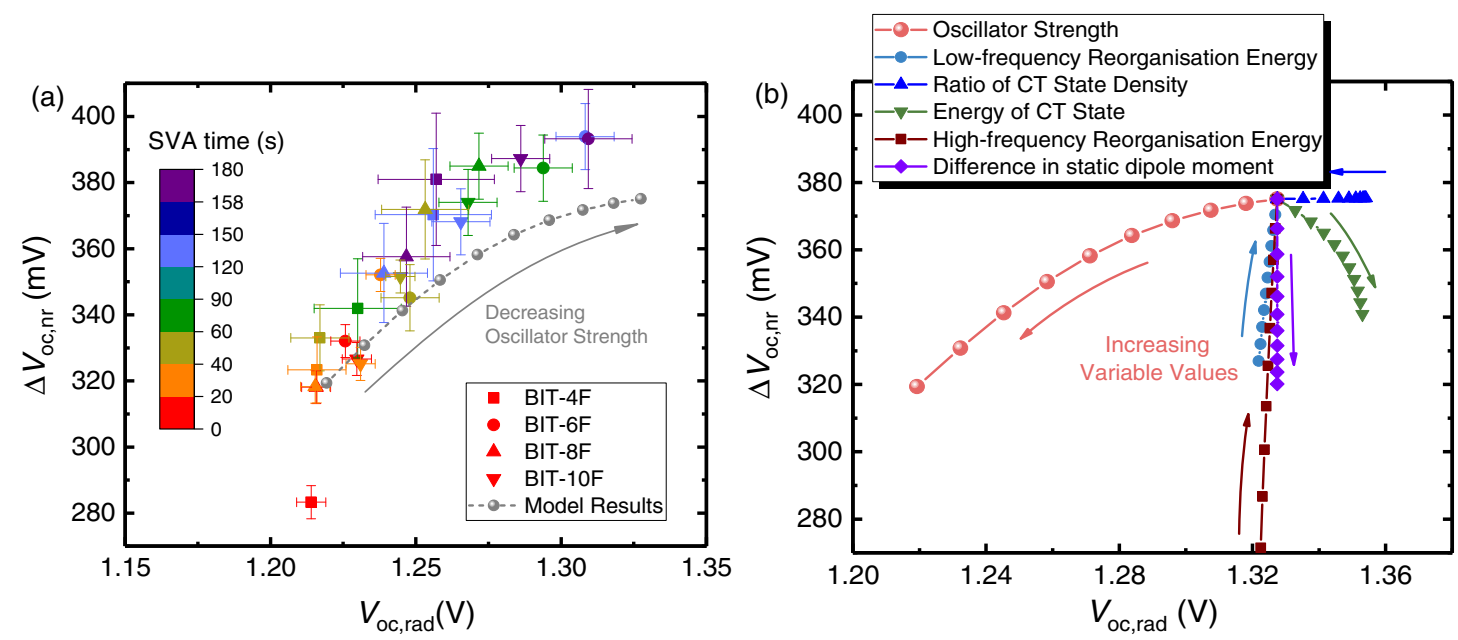

FIG. 4. (a) Calculated (experimental) nonradiative voltage losses $\left(\Delta V_{\mathrm{oc}, \mathrm{nr}}\right)$ as a function of $V_{\mathrm{oc} \text {,rad }}$ with respect to different exposure times in solvent vapor for BIT-4F-, 6F-, 8F-, and 10F-based fullerene solar cells (colored symbols) and simulated (modeled) results considering solely the change of transition oscillator strength (gray dashed line with solid circles). (b) The effect of the different parameters of the model on the variation of $\Delta V_{\mathrm{oc}, \mathrm{nr}}$ with $V_{\mathrm{oc}, \mathrm{rad}}$. The only parameter leading to a strong positive correlation between the two is the oscillator strength. The arrow represents the direction of increasing the specific variable values. The variable ranges are $f_{\text {osc }}=\left[10^{-2}, 1\right], \lambda_{l}=[0.1,0.2] \mathrm{eV}, R_{\mathrm{CT} / E}=\left[10^{-2}, 10^{-4}\right], E_{\mathrm{CT}}=[1.45,1.55] \mathrm{eV}, \lambda_{v}=[0.1,0.2] \mathrm{eV}$, and $\mid \Delta \overrightarrow{\mu \mid}=[10,30] \mathrm{D}$. 
charge-transfer state, a lower absorption coefficient of these states from a lower density of charge-transfer states in the bulk, or a lower oscillator strength for these states. An increase in the energy of the charge-transfer state would decrease $\Delta V_{\text {oc,nr }}$ according to the results of our model, which is inconsistent with the data shown in Fig. 4(a). On the other hand, if we consider a change in the oscillator strength of the charge-transfer states, we can reproduce the same trend as shown in Fig. 4(a) using the model. A summary of how a change in each parameter of the model would affect the relationship between $V_{\text {oc,rad }}$ and $\Delta V_{\text {oc,nr }}$ is presented in Fig. 4(b). This figure shows that the trend whereby $\Delta V_{\text {oc,nr }}$ increases with increasing $V_{\text {oc,rad }}$ as shown in Fig. 4(a) is reproduced only by a reduction in the CT-state oscillator strength.

In order to reproduce the experimental data, we first aim to reconstruct the EQE data using the model by fixing both the band-gap energy and the CT-state energy. Figure 3(b) shows the results of this model using the parameters values from Table I. The values for $E_{\mathrm{CT}}$ and $E_{g}$ are chosen to agree with the experimental parameters, $\lambda_{v}, \lambda_{l}, \hbar \Omega$, and $n$ are chosen as representative of the values reported in the literature for similar systems, while $\mid \Delta \overrightarrow{\mu \mid}$ and the range of $f_{\text {osc }}$ values are chosen to reproduce the values of the radiative and nonradiative voltage losses observed experimentally. Using these data, we calculate both $V_{\text {oc,rad }}$ and $\Delta V_{\text {oc,nr }}$. The dashed line with solid circles in Fig. 4(a) shows how the model can reproduce the experimental data with the assumption that the only parameter changing is the oscillator strength of the transition. The effect of the physical arrangement of the donor and the acceptor at the interface on the CT-state properties have been presented in previous studies [49-52], and it has been shown that increasing the size of the clusters affects both the energy of the CT state and the electronic coupling between the CT state and the ground state. In light of these previous studies, we consider that the reduction in the oscillator strength of the transition upon SVA is related to increased spatial delocalization of the interfacial state. While the ratio of CT- to exciton-state density may be expected to vary upon

TABLE I. Parameter values used to model the experimental data.

\begin{tabular}{lc}
\hline \hline Parameter & Value \\
\hline Difference in Gibbs free energy $\left(\boldsymbol{E}_{\boldsymbol{C T}}\right)$ & $1.45 \mathrm{eV}$ \\
Difference in the static dipole moment $(|\boldsymbol{\Delta} \overrightarrow{\boldsymbol{\mu}}|)$ & $10 \mathrm{D}$ \\
Oscillator strength $\left(\boldsymbol{f}_{\text {osc }}\right)$ & 1 to $1 \times 10^{-3}$ \\
High-frequency reorganization energy $\left(\lambda_{\boldsymbol{v}}\right)$ & $0.2 \mathrm{eV}$ \\
Low-frequency reorganization energy $\left(\lambda_{\boldsymbol{l}}\right)$ & $0.2 \mathrm{eV}$ \\
Vibrational mode harmonic-oscillator energy $(\hbar \boldsymbol{\Omega})$ & $0.15 \mathrm{eV}$ \\
Refractive index $(\boldsymbol{n})$ & 1.5 \\
The ratio of CT-state density $\left(\boldsymbol{R}_{\boldsymbol{C T} / \boldsymbol{E}}\right)$ & 0.01 \\
Band-gap energy $\left(\boldsymbol{E}_{\boldsymbol{g}}\right)$ & $1.75 \mathrm{eV}$ \\
\hline \hline
\end{tabular}

annealing, this ratio will affect radiative and nonradiative processes equally and cannot explain the trend observed.

The same experiment with different SVA times is carried on another small molecule, $p$-DTS $\left(\mathrm{FBTTh}_{2}\right)_{2}$, blended with $\mathrm{PC}_{71} \mathrm{BM}$ [53]. The results shown in Fig. S8 [29] show that the trend for the BIT series is reproduced; therefore, the observed trend is not unique for the BIT-based systems.

These experimental results show that a unique correlation between $\Delta V_{\text {oc,nr }}$ and $V_{\text {oc,rad }}$ is not necessary (or general), in contrast to the suggestion by previous studies $[5,12]$, but the trend depends on other parameters as shown in Fig. 4(b). Therefore, one cannot rely on achieving a change in $V_{\text {oc }}$ simply by varying $\Delta V_{\text {oc,nr }}$ or $\Delta V_{\text {oc,abs }}$ alone. Moreover, the results suggest that $\Delta V_{\text {oc,nr }}$ could be reduced without the compromise to the photocurrent that would result from requiring a larger CT-state energy, in contrast to the conclusion in Ref. [12].

\section{DISCUSSION}

\section{A. Strategies to improve $V_{\text {oc }}$}

We establish a way to quantify one of the main sources of nonradiative voltage losses in bulk heterojunction organic solar cells and relate it to characteristics of the chargetransfer transition. Using the model, we establish what we should aim for to reduce $\Delta V_{\mathrm{oc}, \mathrm{nr}}$. However, as we have noticed, experimentally decreasing $\Delta V_{\mathrm{oc}, \mathrm{nr}}$ can induce an increase of $\Delta V_{\text {oc,abs }}$, leading to nearly stabilized $V_{\mathrm{oc}}$. This correlation complicates the dependence of $V_{\mathrm{oc}}$ on the parameters of the model. Therefore, to improve $V_{\text {oc }}$, one should not focus solely on decreasing $\Delta V_{\mathrm{oc}, \mathrm{nr}}$.

Upon exploring the effect of the different parameters introduced in this work on $V_{\mathrm{oc}}$, we can identify and rank the opportunities to raise $V_{\mathrm{oc}}$. As the effect of different parameters is entangled, we explore their effect in acceptable ranges presented in Table S2 [29]. Starting from a cell with the lowest oscillator strength and parameters as given in Table I, we calculate $V_{\text {oc }}$ by changing each parameter separately in the order presented in Table S2 [29]. From all the combinations, we calculate the average increase of $V_{\text {oc }}$ by changing one parameter. Table II summarizes the potential gain that could be achieved by varying some of the parameters involved in the calculation of $V_{\mathrm{oc}}$ within the ranges given.

The best route to improve $V_{\text {oc }}$ from Table II seems to be a decrease of the reorganization energy associated with the charge-transfer transition. If we find a way to reduce the conformational change of the molecule upon the chargetransfer transition as well as its effect on the surrounding environment, we can improve $V_{\text {oc }}$ by almost $0.2 \mathrm{~V}$. The effect of the charge-transfer energy and the driving force $\left(E_{g}-E_{\mathrm{CT}}\right)$ is also significantly high. When we account for its effect on both the radiative and nonradiative voltage loss, the open-circuit voltages increase by more than the gain due to the energy increase as reported by Benduhn et al. 
TABLE II. Potential open-circuit voltage increase when changing different parameters in the range shown.

\begin{tabular}{lc}
\hline \hline Parameter improvement strategy & $V_{\text {oc }}$ increase \\
\hline Reduce the ratio of CT-state density from $1 \%$ to $0.01 \%$ & $40 \mathrm{mV}$ \\
Reduce the low-frequency reorganization energy $\left(\lambda_{0}\right)$ from 0.2 to $0.1 \mathrm{eV}$ & $55 \mathrm{mV}$ \\
Reduce the high-frequency reorganization energy $\left(\lambda_{i}\right)$ from 0.2 to $0.1 \mathrm{eV}$ & $130 \mathrm{mV}$ \\
Increasing the energy of the CT state by $100 \mathrm{meV}$ (from 1.45 to $1.55 \mathrm{eV})$ & $130 \mathrm{mV}$ \\
Reducing $E_{g}-E_{\mathrm{CT}}$ from 300 to $100 \mathrm{meV}\left(E_{g}\right.$ is decreased) & $140 \mathrm{mV}$ \\
Increasing the oscillator strength of the transition from $1 \times 10^{-2}$ to 1 & $10 \mathrm{mV}$ \\
Increasing the difference in the static dipole moment from 10 to $30 \mathrm{D}$ & $45 \mathrm{mV}$ \\
\hline \hline
\end{tabular}

[12]. The effect of the difference in the static dipole moment of the two states is significantly important, as it can increase $V_{\text {oc }}$ by more than $40 \mathrm{mV}$ just by increasing it within $20 \mathrm{D}$. The effect of the ratio of CT-state density is the least significant. Reducing the density of interfacial states by 2 orders of magnitude would only increase $V_{\text {oc }}$ by $40 \mathrm{mV}$.

Moreover, although not shown by the data in the table, it is straightforward to show that a significant change in the oscillator strength does not affect $V_{\text {oc }}$ in the case of high $E_{g}-E_{\mathrm{CT}}$ systems as demonstrated in the BIT case above with driving forces larger than $0.3 \mathrm{eV}$, where $\Delta V_{\mathrm{oc}, \mathrm{nr}}$ and $\Delta V_{\text {oc,rad }}$ are compensated with each other. However, in the case where the driving force is lower than $0.1 \mathrm{eV}$, the effect of the oscillator strength change on $V_{\text {oc,rad }}$ is negligible [54], as indicated in the EQE shape illustrated in Fig. S9 [29], where we can clearly see the difference between large and small driving-force cases on the effects of the oscillator strength of the CT state. Since increasing the oscillator strength of the CT state always decreases $\Delta V_{\text {oc,nr }}$ according to Eq. (13), the difference of the effect of the oscillator strength of the CT state on $V_{\text {oc,rad }}$ determines the final $V_{\text {oc }}$. Within the open-circuit voltages calculated above, the average increase of $V_{\mathrm{oc}}$ when changing the oscillator strength from $10^{-2}$ to 1 for a $0.1-\mathrm{eV}$ driving force is $64 \mathrm{mV}$, where in the case of $0.3 \mathrm{eV}$ it is as low as $10 \mathrm{mV}$. The impact of CT-state absorptance on solar cell performance was modeled previously using a more phenomenological approach in Ref. [54].

Recent progress in polymer-nonfullerene materials has shown that high performances can be achieved with a lowenergy offset between absorption onset and CT-state energies [5,43]. Therefore, to improve $V_{\mathrm{oc}}$ for these systems, we should aim at improving the emission properties of the charge-transfer state. Moreover, we suspect that the high oscillator strength and strong luminescence efficiency of the best-studied nonfullerene acceptors (NFAs) could explain their better performance as compared to fullerene acceptors. This hypothesis can be rationalized by understanding that the oscillator strength of the chargetransfer state is enhanced by a degree of hybridization with, and intensity borrowing from, the nearest donor or acceptor excited state when the two states are close in energy [37], and, moreover, the CT-state oscillator strength depends upon the oscillator strengths of the singlet states that formed it [55]. Thus, polymer-NFA blends would benefit from the details of the theory discussed here in several ways. First, they enable smaller energy-level offsets leading to higher-energy CT states for a given absorption onset, which leads to lower nonradiative recombination due to the higher number of vibrational modes necessary for a nonradiative transition as well as leading to a higher CT-state oscillator strength as explained above. In addition, blends with NFAs may also benefit from the higher oscillator strength of the acceptor, and hence of the CT state, and the subsequent gain in $V_{\text {oc }}$. One cost of NFA-based solar cells is that the higher CT-state oscillator strengths that lead to higher $V_{\mathrm{oc}}$ 's may also accelerate the total recombination kinetics. While this is no problem at an open circuit, where only the ratios of the rates are important, faster kinetics may be an issue at $V<V_{\text {oc }}$ by imposing more challenging requirements on the mobility to achieve efficient collection. This issue may explain why reports on high-fill-factor devices at active layer thicknesses substantially larger than $100 \mathrm{~nm}$ are so far quite rare $[3,56]$.

\section{B. Efficiency limit for organic solar cell}

In the previous section, we study the importance of the nonradiative recombination process enhanced by the vibrational modes of the states. Using the reported properties of the charge-transfer state to ground-state transition, we are able to account for most of the nonradiative voltage losses in organic solar cell. Thus, the nonradiative losses in OPVs are likely to be dominated by this process. Therefore, we should account for these losses to calculate a theoretical limit for the PCE of an ideal bulk heterojunction organic solar cell.

Using the detailed balance theory, several groups have investigated the radiative limit of organic solar cells $[6,57,58]$. To account for the nonradiative losses, they consider a fixed luminescence quantum yield, showing the increase of the PCE with an improved luminescence yield. In this work, as in the work of Benduhn et al. [12], we establish a relation between the energies of the chargetransfer state and $\Delta V_{\mathrm{oc}, \mathrm{nr}}$. Therefore, using this model, we 
can establish a more realistic efficiency limit for OPVs considering these effects. Benduhn et al. [12] calculate a limit using the extracted parameters from fitting their experimental data, whereas in this work we consider a limit related to the best parameters considered in the model. Moreover, the limit considered in this work takes the excess radiative losses induced by the optical absorption of the charge-transfer state into account.

Considering the best parameters from Table II, we can establish a limit for the $V_{\mathrm{oc}}$ of organic solar cells as a function of the band-gap energy of the absorber material. For this purpose, we consider that $E_{g}=E_{\mathrm{CT}}+0.1$, which is consistent with a number of reports of low-offset systems that still result in high photocurrent quantum efficiency [5,43,59-61]. By using the calculated EQE based on the optical model and the AM1.5G solar spectrum, we estimate a limiting value of $J_{\mathrm{sc}}$. Considering the ideal Shockley diode equation, we estimate the efficiency limit for OPVs considering the limitation induced by the charge-transfer states which is presented as the "nonradiative limit" in Fig. 5. Figure 5 presents the efficiency limit of OPVs from this model as well as a more realistic limit where we consider a limit of $90 \%$ of EQE and $80 \%$ FF. We notice that, due to the high nonradiative losses of organic solar cells, their efficiency is limited to approximately $25 \%$ even in the ideal case and to approximately $20 \%$ for a more realistic case. The optimum band-gap energy of the absorber is blueshifted to around $1.6 \mathrm{eV}$, compared to $1.37 \mathrm{eV}$ for the radiative limit. These results are very similar to those reported in Ref. [12].

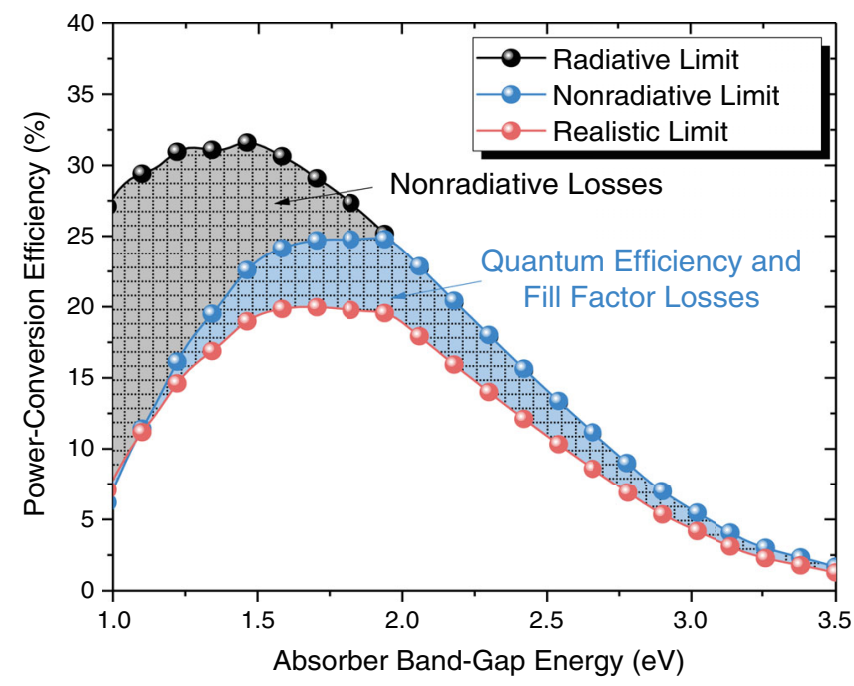

FIG. 5. Predicted power-conversion efficiency compared with the radiative limit. The black line is the SQ limit using the AM1.5G sun spectrum, where only radiative recombination is considered; the blue line is the nonradiative limit considering a perfect charge collection (100\% of the EQE calculated from modeled absorption spectra) and the ideal Shockley equation (ideality factor of 1); the red line is the more realistic limit assuming $90 \%$ EQE absorption and $80 \%$ fill factor.

\section{CONCLUSION}

We presented a model that quantifies the nonradiative voltage losses in OPV due to nonradiative recombination through the charge-transfer state. We established the different parameters affecting its value which help improve our understanding of the phenomena and the limitation facing OPV devices due to these interfacial states.

From the experimental data on a series of (BIT) oligomer donors blended with $\mathrm{PC}_{71} \mathrm{BM}$, we emphasized that the change in $E_{\mathrm{CT}}$ cannot explain the results, and therefore there must have been other parameters to consider. From the effect of SVA on these small molecules, we noticed a linear trend between $\Delta V_{\mathrm{oc}, \mathrm{nr}}$ and $V_{\mathrm{oc}, \text { rad }}$ that conserves $V_{\mathrm{oc}}$. The observed trend was different from that previously reported [5,12]. In order to explain this observation, we studied the effect of the different parameters of the model on $\Delta V_{\text {oc,nr }}$ and $V_{\text {oc,rad }}$ and established that the change in the oscillator strength presents a good explanation for the experimental results. We also established that the increase of $\Delta V_{\text {oc,nr }}$ did not affect $V_{\text {oc }}$, as the increase was compensated by a decrease of the absorption broadening losses. This finding emphasizes that in order to increase $V_{\text {oc }}$ one should separately address and attempt to minimize both types of voltage loss $\left(\Delta V_{\text {oc,nr }}\right.$ and $\left.\Delta V_{\text {oc,abs }}\right)$.

We explored the effect of the different parameters on $V_{\mathrm{oc}}$ and ranked the routes to improve it. We have shown that reducing both reorganization energies presents the best opportunity to increase $V_{\mathrm{oc}}$ and, therefore, the power-conversion efficiency of the cells. By exploring the efficiency limit of OPVs due to the essential role of the charge-transfer state in charge generation and recombination, we established that OPV efficiencies can reach values close to $25 \%$ in the ideal case, with a more realistic limit at 20\%. Therefore, this result emphasizes the potential of this technology to compete with other inorganic photovoltaic technologies.

By relating the voltage losses to different properties of the donor and acceptor molecules, and particularly to the CT-state oscillator strength, this work helps to unveil the origin of the recent improvement in performance achieved by nonfullerene acceptor devices [62]. An approach related solely to the energy levels of the donor, acceptor, and their CT state would not explain the differences in open-circuit voltage behavior between devices based on nonfullerene acceptors and those based on fullerenes.

Many parameters of the model are hard to control. A better understanding of what affects these parameters is necessary if we aim to improve the open-circuit voltage of these cells and reach higher efficiencies. Moreover, this model considers only one type of nonradiative recombination mechanism. In the limit where this recombination is not dominant, we must include the effect of other recombination processes such as trap-assisted recombination or surface recombination at the contacts. 


\section{METHODS}

\section{A. Organic solar cell fabrication}

The device structure in this study is ITO/PEDOT: PSS/small-molecule: $\mathrm{PC}_{71} \mathrm{BM} / \mathrm{PFN} / \mathrm{Al}$, where poly(9,9bis(3'-(N,N-dimethylamino)propyl)-2,7-fluorene)-alt-2,7(9,9-dioctylfluorene) (PFN) and poly(3,4-ethylenedioxythiophene):poly(styrenesulfonate) (PEDOT:PSS) (Clevios PPVP AI 4083, H.C Starck Inc.) were employed as the cathode and anode buffer layer, respectively. A 40-nmthick PEDOT:PSS anode buffer layer was spin-cast on the precleaned ITO anode substrate and then dried in air at $150^{\circ} \mathrm{C}$ for $20 \mathrm{~min}$. Subsequently, a thin layer of smallmolecule: $\mathrm{PC}_{71} \mathrm{BM}$ blend was deposited atop the PEDOT: PSS/ITO substrate by spin-coating with a hot chlorobenzene solution, followed by solvent-annealing treatment in a Petri dish containing $2-3 \mathrm{~mL} \mathrm{CH}_{2} \mathrm{Cl}_{2}$. Soon after the treatment, a 5-nm PFN layer was spin-coated from methanol solution in the presence of a trace amount of acetic acid onto the active layer. Finally, the films were transferred into a vacuum evaporator, and $70-100 \mathrm{~nm}$ of $\mathrm{Al}$ are deposited as the cathode.

\section{B. Current density versus voltage $(\boldsymbol{J}-\boldsymbol{V})$ characteristics}

The values of power-conversion efficiency were determined from $J-V$ characteristics measured by a Keithley 2400 source-measurement unit under the AM 1.5G spectrum from a solar simulator (Oriel Instruments).

\section{Electroluminescence}

The measurement was measured using a Shamrock 303 spectrograph combined with an iDUS InGaAs array detector cooled to $-90^{\circ} \mathrm{C}$. The driving injection current is in the range of $1.25-1250 \mathrm{~mA} / \mathrm{cm}^{2}$. The obtained EL spectra intensity was calibrated with the spectrum from a calibrated halogen lamp.

\section{External quantum efficiency}

The measurement was measured using a grating spectrometer (CS260-RG-4-MT-D) to create monochromatic light combined with a tungsten halogen light source. The monochromatic light was chopped at approximately $300 \mathrm{~Hz}$, and a Stanford Research Systems SR380 lockin amplifier with an internal transimpedance amplifier of $10^{6} \mathrm{~V} / \mathrm{A}$ was used to detect the photocurrent. Long-pass filters at $610,715,780,850$, and $1000 \mathrm{~nm}$ were used to filter out the scattered light from the monochromator. The spectra were taken from 300 to $1100 \mathrm{~nm}$ and calibrated by a silicon photodiode.

\section{ACKNOWLEDGMENTS}

M. A. thanks the ESPRC for support. J. N. has received funding from the EPSRC (Grants No. EP/P005543/1 and
No. EP/M025020/1), the EPSRC Supersolar Hub (EP/ P02484X/1), and the European Research Council under the European Union's Horizon 2020 research and innovation program (Grant Agreement No. 742708). J. Y. and H. W. thank the National Nature Science Foundation of China (No. 51225301, No. 21472012, No. 51403066, No. 51521002, No. 91333206, and No. 5141101251). J. Y. acknowledges the support from China Scholarship Council (CSC). The authors thank Xingyuan Shi, Jason Rohr, Flurin Eisner, and Sachetan M. Tuladhar for fruitful discussions. T. K. acknowledges support from the DFG (Grant No. KI-1571/2-1).

[1] J. Hou, O. Inganäs, R. H. Friend, and F. Gao, Organic Solar Cells Based on Non-Fullerene Acceptors, Nat. Mater. 17, 119 (2018).

[2] H. Li, Z. Xiao, L. Ding, and J. Wang, Thermostable SingleJunction Organic Solar Cells with a Power Conversion Efficiency of 14.62\%, Science bulletin 63, 340 (2018).

[3] T. Kirchartz, P. Kaienburg, and D. Baran, Figures of Merit Guiding Research on Organic Solar Cells, J. Phys. Chem. C 122, 5829 (2018).

[4] C. Yan, S. Barlow, Z. Wang, H. Yan, A. K. Y. Jen, S. R. Marder, and X. Zhan, Non-Fullerene Acceptors for Organic Solar Cells, Nat. Rev. Mater. 3, 18003 (2018).

[5] D. Baran, T. Kirchartz, S. Wheeler, S. Dimitrov, M. Abdelsamie, J. Gorman, R. S. Ashraf, S. Holliday, A. Wadsworth, N. Gasparini, P. Kaienburg, H. Yan, A. Amassian, C. J. Brabec, J. R. Durrant, and I. McCulloch, Reduced Voltage Losses Yield 10\% Efficient Fullerene Free Organic Solar Cells with > 1 V Open Circuit Voltages, Energy Environ. Sci. 9, 3783 (2016).

[6] K. Vandewal, K. Tvingstedt, A. Gadisa, O. Inganäs, and J. V. Manca, Relating the Open-Circuit Voltage to Interface Molecular Properties of Donor:Acceptor Bulk Heterojunction Solar Cells, Phys. Rev. B 81, 125204 (2010).

[7] J. Benduhn, F. Piersimoni, G. Londi, A. Kirch, J. Widmer, C. Koerner, D. Beljonne, D. Neher, D. Spoltore, and K. Vandewal, Impact of Triplet Excited States on the OpenCircuit Voltage of Organic Solar Cells, Adv. Energy Mater. 8, 1800451 (2018).

[8] C. Deibel, A. Wagenpfahl, and V. Dyakonov, Origin of Reduced Polaron Recombination in Organic Semiconductor Devices, Phys. Rev. B 80, 075203 (2009).

[9] L. J. A. Koster, E. C. P. Smits, V. D. Mihailetchi, and P. W. M. Blom, Device Model for the Operation of Polymer/Fullerene Bulk Heterojunction Solar Cells, Phys. Rev. B 72, 085205 (2005).

[10] D. Bartesaghi, I. del, C. Pérez, J. Kniepert, S. Roland, M. Turbiez, D. Neher, and L. J. A. Koster, Competition between Recombination and Extraction of Free Charges Determines the Fill Factor of Organic Solar Cells, Nat. Commun. 6, 7083 (2015).

[11] T. M. Burke, S. Sweetnam, K. Vandewal, and M. D. McGehee, Beyond Langevin Recombination: How Equilibrium between Free Carriers and Charge Transfer States 
Determines the Open-Circuit Voltage of Organic Solar Cells, Adv. Energy Mater. 5, 1500123 (2015).

[12] J. Benduhn, K. Tvingstedt, F. Piersimoni, S. Ullbrich, Y. Fan, M. Tropiano, K. A. McGarry, O. Zeika, M. K. Riede, C. J. Douglas, S. Barlow, S. R. Marder, D. Neher, D. Spoltore, and K. Vandewal, Intrinsic Non-radiative Voltage Losses in Fullerene-Based Organic Solar Cells, Nat. Energy 2, 17053 (2017).

[13] M. Bixon, J. Jortner, J. Cortes, H. Heitele, and M. E. Michel-Beyerle, Energy Gap Law for Nonradiative and Radiative Charge Transfer in Isolated and in Solvated Supermolecules, J. Phys. Chem. 98, 7289 (1994).

[14] R. Englman and J. Jortner, The Energy Gap Law for Radiationless Transitions in Large Molecules, Mol. Phys. 18, 145 (1970).

[15] A. Nitzan, S. Mukamel, and J. Jortner, Energy Gap Law for Vibrational Relaxation of a Molecule in a Dense Medium, J. Chem. Phys. 63, 200 (1975).

[16] B. K. Ridley, Multiphonon, Non-radiative Transition Rate for Electrons in Semiconductors and Insulators, J. Phys. C 11, 2323 (1978).

[17] B. K. Ridley, On the Multiphonon Capture Rate in Semiconductors, Solid State Electron. 21, 1319 (1978).

[18] B. K. Ridley, Quantum Processes in Semiconductors (Oxford University, New York, 2013).

[19] T. Markvart, Semiclassical Theory of Non-radiative Transitions, J. Phys. C 14, L895 (1981).

[20] W. Shockley and H. J. Queisser, Detailed Balance Limit of Efficiency of p-n Junction Solar Cells, J. Appl. Phys. 32, 510 (1961).

[21] J. Yao, T. Kirchartz, M. S. Vezie, M. A. Faist, W. Gong, Z. He, H. Wu, J. Troughton, T. Watson, D. Bryant, and J. Nelson, Quantifying Losses in Open-Circuit Voltage in Solution-Processable Solar Cells, Phys. Rev. Applied 4, 014020 (2015).

[22] U. Rau, U. W. Paetzold, and T. Kirchartz, Thermodynamics of Light Management in Photovoltaic Devices, Phys. Rev. B 90, 035211 (2014).

[23] J. Reinhardt, M. Grein, C. Bühler, M. Schubert, and U. Würfel, Identifying the Impact of Surface Recombination at Electrodes in Organic Solar Cells by Means of Electroluminescence and Modeling, Adv. Energy Mater. 4, 1400081 (2014).

[24] B. Blank, T. Kirchartz, S. Lany, and U. Rau, Selection Metric for Photovoltaic Materials Screening Based on DetailedBalance Analysis, Phys. Rev. Applied 8, 024032 (2017).

[25] P. F. Barbara, T. J. Meyer, and M. a. Ratner, Contemporary Issues in Electron Transfer Research, J. Phys. Chem. 100, 13148 (1996).

[26] I. R. Gould, R. H. Young, L. J. Mueller, a. C. Albrecht, and S. Farid, Electronic Structures of Exciplexes and Excited Charge-Transfer Complexes, J. Am. Chem. Soc. 116, 8188 (1994).

[27] I. R. Gould, D. Noukakis, J. L. Goodman, R. H. Young, and S. Farid, A Quantitative Relationship between Radiative and Nonradiative Electron Transfer in Radical-Ion Pairs, Chem. Phys. 176, 439 (1993).

[28] J. Jortner, Temperature Dependent Activation Energy for Electron Transfer between Biological Molecules, J. Chem. Phys. 64, 4860 (1976).
[29] See Supplemental Material at http://link.aps.org/ supplemental/10.1103/PhysRevX.8.031055 for the derivation of $k_{r}$, the table for parameter ranges (Table $\mathrm{S} 1$ ), parameter ranges for the $V_{\mathrm{oc}}$ improvement simulation (Table S2), the detailed version of Fig. 2(d) (Fig. S1), the chemical structure of four multi-fluorine substituted oligomers (Fig. S2), device performance for BIT-4F, 6F, 8F, and 10F (Fig. S3-S6), EL and EQE data for BIT-6F, 8F, and 10F (Fig. S7), experimental data for $p$-DTS $\left(\mathrm{FBTTh}_{2}\right)_{2}$ based devices (Fig. S8), simulated EQE spectrum comparison between large and small driving force devices (Fig. S9).

[30] K. Huang and A. Rhys, Theory of Light Absorption and Non-Radiative Transitions in F-Centres, Proc. R. Soc. A 204, 406 (1950).

[31] T. H. Keil, Shapes of Impurity Absorption Bands in Solids, Phys. Rev. 140, A601 (1965).

[32] M. Malagoli, V. Coropceanu, D. A. Da Silva Filho, and J. L. Brédas, A Multimode Analysis of the Gas-Phase Photoelectron Spectra in Oligoacenes, J. Chem. Phys. 120, 7490 (2004).

[33] H. Sumi, in Electron Transfer in Chemistry (Wiley-VCH Verlag GmbH, Weinheim, 2008), pp. 64-108.

[34] H. S. Ren, M. J. Ming, J. Y. Ma, and X. Y. Li, Theoretical Calculation of Reorganization Energy for Electron SelfExchange Reaction by Constrained Density Functional Theory and Constrained Equilibrium Thermodynamics, J. Phys. Chem. A 117, 8017 (2013).

[35] K. Vandewal, J. Benduhn, K. S. Schellhammer, T. Vangerven, J. E. Rückert, F. Piersimoni, R. Scholz, O. Zeika, Y. Fan, S. Barlow, D. Neher, S. R. Marder, J. Manca, D. Spoltore, G. Cuniberti, and F. Ortmann, Absorption Tails of Donor: C60 Blends Provide Insight into Thermally Activated ChargeTransfer Processes and Polaron Relaxation, J. Am. Chem. Soc. 139, 1699 (2017).

[36] D. Bhattacharya, N. Vaval, and S. Pal, Electronic Transition Dipole Moments and Dipole Oscillator Strengths within Fock-Space Multi-reference Coupled Cluster Framework: An Efficient and Novel Approach, J. Chem. Phys. 138, 094108 (2013).

[37] S. Few, J. M. Frost, J. Kirkpatrick, and J. Nelson, Influence of Chemical Structure on the Charge Transfer State Spectrum of a Polymer:Fullerene Complex, J. Phys. Chem. C 118, 8253 (2014).

[38] N.S. Hush, Intervalence-Transfer Absorption. Part 2. Theoretical Considerations and Spectroscopic Data, in Progress in Inorganic Chemistry, edited by F. A. Cotton (Publisher, location, 2007), pp. 391-444.

[39] J. E. Subotnik, S. Yeganeh, R. J. Cave, and M. A. Ratner, Constructing Diabatic States from Adiabatic States: Extending Generalized Mulliken-Hush to Multiple Charge Centers with Boys Localization, J. Chem. Phys. 129, 244101 (2008).

[40] G. C. Schatz and M. A. Ratner, Quantum Mechanics in Chemistry (Courier, Chelmsford, MA, 2002).

[41] R. C. Hilborn, Einstein Coefficients, Cross Sections, $f$ Values, Dipole Moments, and All That, Am. J. Phys. 50, 982 (1982).

[42] S. M. Tuladhar, M. Azzouzi, F. Delval, J. Yao, A. A. Y. Guilbert, T. Kirchartz, N. F. Montcada, R. Dominguez, F. Langa, E. Palomares, and J. Nelson, Low Open-Circuit 
Voltage Loss in Solution-Processed Small-Molecule Organic Solar Cells, ACS Energy Lett. 1, 302 (2016).

[43] J. Liu, S. Chen, D. Qian, B. Gautam, G. Yang, J. Zhao, J. Bergqvist, F. Zhang, W. Ma, H. Ade, O. Inganäs, K. Gundogdu, F. Gao, and H. Yan, Fast Charge Separation in a Non-Fullerene Organic Solar Cell with a Small Driving Force, Nat. Energy 1, 16089 (2016).

[44] Z. Fei, F. D. Eisner, X. Jiao, M. Azzouzi, J. A. Röhr, Y. Han, M. Shahid, A. S. R. Chesman, C. D. Easton, C. R. McNeill, T. D. Anthopoulos, J. Nelson, and M. Heeney, An Alkylated Indacenodithieno[3,2- b] thiophene-Based Nonfullerene Acceptor with High Crystallinity Exhibiting Single Junction Solar Cell Efficiencies Greater than 13\% with Low Voltage Losses, Adv. Mater. 30, 1705209 (2018).

[45] J. L. Wang, K. K. Liu, J. Yan, Z. Wu, F. Liu, F. Xiao, Z. F. Chang, H. Bin Wu, Y. Cao, and T. P. Russell, Series of Multifluorine Substituted Oligomers for Organic Solar Cells with Efficiency over 9\% and Fill Factor of 0.77 by Combination Thermal and Solvent Vapor Annealing, J. Am. Chem. Soc. 138, 7687 (2016).

[46] J. Yan, Q. Liang, K. Liu, J. Miao, H. Chen, S. Liu, Z. He, H. Wu, J. Wang, and Y. Cao, Optimized Phase Separation and Reduced Geminate Recombination in High Fill Factor Small-Molecule Organic Solar Cells, ACS Energy Lett. 2, 14 (2017).

[47] K. R. Graham, C. Cabanetos, J. P. Jahnke, M. N. Idso, A. El Labban, G. O. Ngongang Ndjawa, T. Heumueller, K. Vandewal, A. Salleo, B. F. Chmelka, A. Amassian, P. M. Beaujuge, and M. D. McGehee, Importance of the Donor: Fullerene Intermolecular Arrangement for High-Efficiency Organic Photovoltaics, J. Am. Chem. Soc. 136, 9608 (2014).

[48] J. Min, X. Jiao, I. Ata, A. Osvet, T. Ameri, P. Bäuerle, H. Ade, and C. J. Brabec, Time-Dependent Morphology Evolution of Solution-Processed Small Molecule Solar Cells during Solvent Vapor Annealing, Adv. Energy Mater. 6, 1502579 (2016).

[49] J. L. Brédas, D. Beljonne, V. Coropceanu, and J. Cornil, Charge-Transfer and Energy-Transfer Processes in $\pi$-Conjugated Oligomers and Polymers: A Molecular Picture, Chem. Rev. 104, 4971 (2004).

[50] X. Shen, G. Han, and Y. Yi, The Nature of Excited States in Dipolar Donor/Fullerene Complexes for Organic Solar Cells: Evolution with the Donor Stack Size, Phys. Chem. Chem. Phys. 18, 15955 (2016).

[51] X.-K. Chen, M. K. Ravva, H. Li, S. M. Ryno, and J.-L. Brédas, Effect of Molecular Packing and Charge Delocalization on the Nonradiative Recombination of ChargeTransfer States in Organic Solar Cells, Adv. Energy Mater. 6, 1601325 (2016).

[52] X. Liu, K. Ding, A. Panda, and S. R. Forrest, Charge Transfer States in Dilute Donor-Acceptor Blend Organic Heterojunctions, ACS Nano 10, 7619 (2016).
[53] T. S. van der Poll, J. A. Love, T.-Q. Nguyen, and G. C. Bazan, Non-Basic High-Performance Molecules for Solution-Processed Organic Solar Cells, Adv. Mater. 24, 3646 (2012).

[54] L. J. A. Koster, S. E. Shaheen, and J. C. Hummelen, Pathways to a New Efficiency Regime for Organic Solar Cells, Adv. Energy Mater. 2, 1246 (2012).

[55] D. Qian, Z. Zheng, H. Yao, W. Tress, T. R. Hopper, S. Chen, S. Li, J. Liu, S. Chen, J. Zhang, X.-K. Liu, B. Gao, L. Ouyang, Y. Jin, G. Pozina, I. A. Buyanova, W. M. Chen, O. Inganäs, V. Coropceanu, J.-L. Bredas et al., Design Rules for Minimizing Voltage Losses in HighEfficiency Organic Solar Cells, Nat. Mater. 17, 703 (2018).

[56] B. Guo, W. Li, X. Guo, X. Meng, W. Ma, M. Zhang, and Y. Li, High Efficiency Nonfullerene Polymer Solar Cells with Thick Active Layer and Large Area, Adv. Mater. 29, 1702291 (2017).

[57] M. Gruber, J. Wagner, K. Klein, U. Hörmann, A. Opitz, M. Stutzmann, and W. Brütting, Thermodynamic Efficiency Limit of Molecular Donor-Acceptor Solar Cells and Its Application to Diindenoperylene/C 60-Based Planar Heterojunction Devices, Adv. Energy Mater. 2, 1100 (2012).

[58] T. Kirchartz, K. Taretto, and U. Rau, Efficiency Limits of Organic Bulk Heterojunction Solar Cells, J. Phys. Chem. C 113, 17958 (2009).

[59] A. Tang, B. Xiao, Y. Wang, F. Gao, K. Tajima, H. Bin, Z.-G. Zhang, Y. Li, Z. Wei, and E. Zhou, Simultaneously Achieved High Open-Circuit Voltage and Efficient Charge Generation by Fine-Tuning Charge-Transfer Driving Force in Nonfullerene Polymer Solar Cells, Adv. Funct. Mater. 28, 1704507 (2018).

[60] S. M. Menke, A. Cheminal, P. Conaghan, N. A. Ran, N. C. Greehnam, G. C. Bazan, T.-Q. Nguyen, A. Rao, and R. H. Friend, Order Enables Efficient Electron-Hole Separation at an Organic Heterojunction with a Small Energy Loss, Nat. Commun. 9, 277 (2018).

[61] H. Bin, L. Gao, Z. G. Zhang, Y. Yang, Y. Zhang, C. Zhang, S. Chen, L. Xue, C. Yang, M. Xiao, and Y. Li, $11.4 \%$ Efficiency Non-fullerene Polymer Solar Cells with Trialkylsilyl Substituted 2D-Conjugated Polymer as Donor, Nat. Commun. 7, 13651 (2016).

[62] W. Li, L. Ye, S. Li, H. Yao, H. Ade, and J. Hou, A High-Efficiency Organic Solar Cell Enabled by the Strong Intramolecular Electron Push-Pull Effect of the Nonfullerene Acceptor, Adv. Mater. 30, 1707170 (2018).

Correction: Minor errors were found in Eqs. (8), (15), and (16) and in a few phrases in text from Sec. II and have been fixed. 